\title{
Using "Student Technology" in Introductory Physics: A Comparison of Three Tools to Study Falling Objects
}

Fábio Saraiva da Rocha, Universidade Federal do Pampa, Brazil; Fabio Fajardo, Universidad Nacional de Colombia, Bogotá, Colombia; Maricarmen Grisolía, Universidad de Los Andes, Mérida,Venezuela; Julio Benegas, Universidad Nacional de San Luis, Argentina; Robert Tchitnga, University of Dschang, Cameroon; Priscilla Laws, Dickinson College, Carlisle, PA

B eing able to facilitate effective hands-on laboratory experiences in introductory physics courses is a challenging task, even when contemporary laboratory facilities, equipment, and new technologies for data collection and analysis are available. At institutions without adequate resources, especially those in developing countries, we have found that the problem of providing effective laboratory experiences is especially daunting for at least two reasons: 1 ) the lack of equipment and contemporary measuring devices; and 2) even at institutions that have some laboratory equipment, students who have access to cell phones with digital timing and video capabilities or inexpensive digital cameras are bored with trying to use "old-fashioned" apparatus for measurements.

We are particularly interested in the educational potential of cell phones because using their own cell phones as scientific apparatus should motivate and engage students in active learning activities. Recent TPT articles have shown that a cell phone photograph of a water jet can be used to analyze parabolic motion, ${ }^{3}$ and a ringing cell phone in a vacuum can be used to study sound wave propagation. ${ }^{4}$ In addition, cell phone screens are useful in the study of other topics such as light polarization. ${ }^{5}$ Furthermore, instructors have been taking advantage of new smartphone "apps" to create accelerometer readings and sound demonstrations. ${ }^{6}$

Here we want to consider a potential lab activity for which an instructor has only one computer equipped with a USB port and Logger Pro graphical and video analysis tools ${ }^{7}$ but no other technology. Imagine that the instructor challenges students to use their cell phones or inexpensive digital cameras to predict and then investigate how objects fall with varying degrees of air drag. The main goal of our contribution is to discuss the potential of two different devices, digital cameras and cell phones with video capabilities, as tools for meaningful laboratory work on the study of falling objects. We then compare the results students might obtain to those obtained using a Vernier Go Motion sensor ${ }^{7}$ hooked directly to a computer USB port.

\section{The experiments}

Figure 1 shows the basic experimental setup. Two identical objects of equal mass, consisting of disposable aluminum pie plates $(28 \mathrm{~cm} \mathrm{x} 22 \mathrm{~cm})$, are used. One object is compacted to form a ball of approximately 7 -cm diameter. The objects are

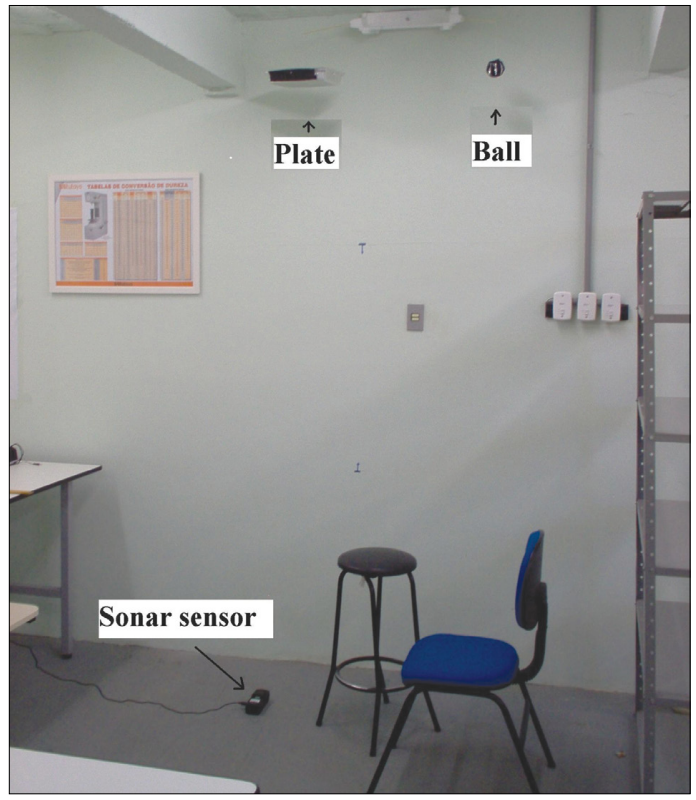

Fig. 1. Experimental setup, as seen from the position of the recording cell phone and digital camera.

released from rest at a height of almost $3 \mathrm{~m}$ while the information needed to obtain position-time data for the falling objects is recorded with: 1) an ultrasonic motion detector ${ }^{7}$ sitting on the floor and connected directly to a computer; 2 ) a cell phone in video mode; and 3 ) with a digital camera in video mode. For this experiment we used two relatively unsophisticated models for the "student" devices-a Sony Ericson Z530i cell phone, recording $15 \mathrm{fps}$, and an HP Photosmart digital camera, which records $30 \mathrm{fps}$. A wooden stick of known length was used as the reference object for scaling. Data handling and analysis were carried out with the Logger Pro 3.6.1 ${ }^{7}$ software, which, besides registering and processing the motion detector data, has video analysis capabilities. The Logger $\mathrm{Pro}^{7}$ software uses numerical derivatives to provide all kinematics graphs (position, velocity, and acceleration versus time) for the data taken by the motion detector. Students can use the Logger Pro video analysis capability to obtain kinematics data and graphs from the two video recordings.

Since video analysis can also be carried out using free software such as Tracker, ${ }^{8}$ the experiments involving video data can be conducted at no cost, in a regular classroom setting without any formal lab space or equipment. Here the motion detector data are shown as a comparison standard, so the in- 


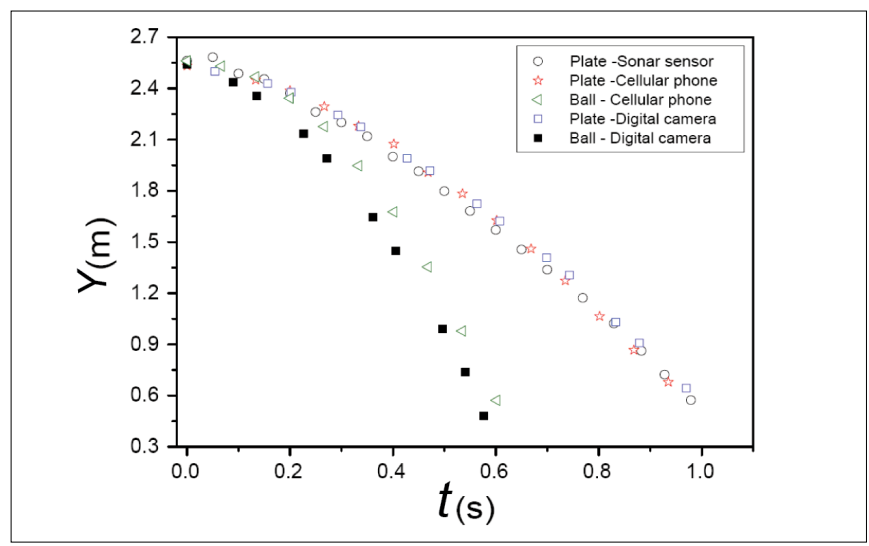

Fig. 2. Graph of position (height) vs time for the plate (extended body) and ball (compacted plate) as determined by the motion detector (sonar) and cellular phone and digital camera video analysis.

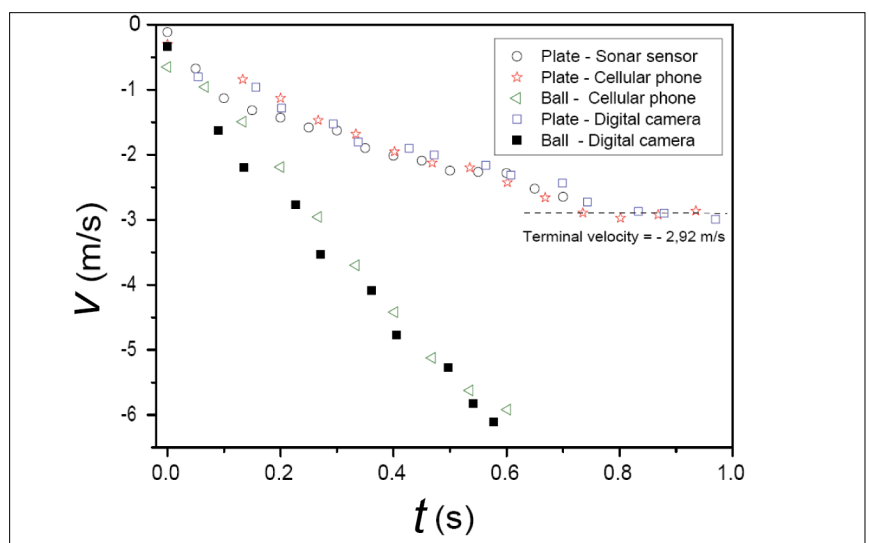

Fig. 3. Velocity-vs-time graphs for the plate (extended body) and ball (compacted plate). Data from the motion detector (sonar), cellular phone, and digital camera. Linear fits to the corresponding data provide the values of terminal velocity and acceleration.

terested instructor can choose the system best suited for his/ her course conditions and objectives.

Figure 2 shows the vertical position-time graphs provided by Logger Pro through motion detector data or by user-directed video analysis for the two falling objects. In all cases the data show the main conceptual features, a parabolic position graph for the compacted aluminum plate, and a graph that is changing from curved at the beginning to a fairly straight line for the extended plate once it reaches terminal velocity. Next the Logger Pro "Analyze" and "Graphing" features were used to display the corresponding velocity-versus-time graphs shown in Fig. 3. Linear fitting was then used to find the terminal velocity of the extended plate $(-2.92 \pm 0.29 \mathrm{~m} / \mathrm{s})$, while a similar fit of cell phone data for the compacted object resulted in a value of $9.57 \pm 0.31 \mathrm{~m} / \mathrm{s}^{2}$ for the acceleration of the compacted body. Comparable results are obtained from the digital camera video data.

Although all three devices provide adequate results, each one presents distinct experimental capabilities, challenges, and difficulties. For instance, the motion detector is rather difficult to focus on the subject, and obtaining reasonable ve- locity-versus-time graphs needed to determine accelerations requires some practice. On the other hand, the video images are blurry when the falling objects are moving fast, producing a time-dependent position uncertainty. As a result the position-versus-time data of the compacted body registered with the cellular phone have a somewhat higher uncertainty as compared with the digital camera, an effect that can be attributed to the fact that the digital camera video is capable of $30 \mathrm{fps}$ while our cell phone video is only capable of $15 \mathrm{fps}$.

\section{An educational outlook}

Does technology by itself improve physics learning? Unfortunately, technology use is often assumed to go hand-in-hand with good teaching and effective learning. Research in physics education has shown time and again the critical importance of using a learning process that requires students' active involvement in order to achieve significant student learning gains. ${ }^{1}$ Therefore, we believe that instructors should concentrate on how technologies can be used as effective tools to facilitate the learning process. These objectives are consistent with those set for laboratory work by others. ${ }^{9-11}$ Five different roles or goals of the physics laboratory have been pointed by a rather recent document from AAPT: ${ }^{9}$ developing the art of experimentation, fostering experimental and analytical skills, contributing to conceptual learning, understanding the basics of physics knowledge, and developing collaborative learning skills. All of these goals, surely to different levels depending on the characteristics of the physics course, could be included in the present experiment. For instance, students could be asked to design the experiment and investigate the best way to position the cell phone or camera and lights to minimize possible parallax errors. By comparing data from different videos, students can also perform in-depth studies of their uncertainties related to the quality of their images as a function of their moving object speeds and camera shutter speeds. Using this type of analysis, they can decide, as we did in the present example, whether or not the digital camera is a more appropriate device than the cell phone. They can also experiment with Logger Pro data smoothing options and discuss how to obtain better acceleration graphs.

Determining the equipment needed to achieve a given uncertainty or error should help students acquire measurement and data handling skills and at the same time foster conceptual learning. For instance, they can analyze whether or not the data are precise enough to discriminate between two different models, e.g., is the effect of air better described by a retarding force proportional to velocity or to the square of the velocity? Thus, modeling and conceptual learning can easily be introduced as laboratory goals, as well as data processing and fitting. Refinements in data analysis can also be used to discuss how well the behavior of the compacted body approximates that of a point mass. Since some small air drag can be noticed from our compacted object data, students can deduce that the acceleration of the compact object is changing.

Last but not least, since all three techniques demand team- 
work, our explorations exemplify how collaborative learning can be introduced into laboratory projects that invite students to compare alternative measurement techniques.

But whatever objectives the instructor defines for laboratory work in a given topic, it should be stressed that satisfactory learning gains always require students' involvement in their own learning process. In this active learning approach, it is fundamental that each student be asked, before carrying out an investigation, to express his or her own views of the physical phenomena, discuss them with peers, eventually compare common predictions with the data they obtained, and resolve discrepancies. If readily available, high-tech devices such as cell phones and digital cameras are used within this educational context, and they can become powerful tools for vitally needed science education reform in our developing countries.

\section{Acknowledgments}

We are grateful to the International Centre for Theoretical Physics (ICTP) of Trieste, Italy, for the generous support that made possible our participation in the Physware ${ }^{12}$ workshop, where the work was initiated. We appreciate the guidance of all workshop facilitators, and especially thank Prof. Priscilla Laws for encouraging us to refine our measurements and prepare this article.

\section{References}

1 Edward F. Redish, Teaching Physics with the Physics Suite (Wiley, Hoboken, NJ, 2004).

2. Priscilla W. Laws, "Millikan Lecture 1996: Promoting active learning based on physics education research in introductory physics courses," Am. J. Phys. 65, 14-21 (1997).

3. A. E. G Falcão Jr., R. A. Gomes, J. M.Pereira, L. F. S. Coelho, and A. C. F Santos. "Cellular phones helping to get a clearer picture of kinematics," Phys. Teach. 47, 167-168 (March 2009).

4. Christian Villa, "Bell-jar demonstration using cell phones," Phys. Teach. 47, 59 (Jan. 2009).

5. Dave Van Domelen, "Teaching light polarization with cell phones," Phys. Teach. 45, 467-469 (Nov. 2007).

6. Douglas Brown and Anne J. Cox, "Innovative uses of video analysis," Phys. Teach. 47, 145-150 (March 2009).

7. Vernier Software \& Technology; www.vernier.com/.

8. Tracker software; www.cabrillo.edu/ dbrown/tracker/.

9. AAPT, "Goals of the introductory physics laboratory," Am. J. Phys. 66, 483-485 (June 1998).

10. Christina Hart, Pamela Mulhall, Amanda Berry, John Loughran, and Richard Gunstone, "What is the purpose of this experiment? Or can students learn from doing experiments?" J. Res. Sci. Teach. 37(7), 655-675 (2000).

11. E. Etkina, A. Van Heuvelen, D. T. Brookes, and D. Mills, "Role of experiments in physics instruction - A process approach," Phys. Teach. 40, 351-55 (Sept. 2002).

12. Physware workshop, ICTP, Trieste, Italy, 2009; cdsagenda5. ictp.trieste.it/full_display.php?ida=a07137.

\section{Physware Description, by Priscilla Laws}

In February 2009 a group of university-level physics teachers from 27 countries in Africa, Asia, Latin America, and Europe spent two weeks working together at the Abdus Salam International Center for Theoretical Physics (ICTP) in Trieste, Italy. The goal of the course was to help instructors from developing countries learn new ways to teach kinematics and dynamics using active learning techniques based on the outcomes of Physics Education Research, and involving the use of lowcost equipment and technologies. The article in this issue of TPT on the comparison of high-tech tools for studying falling objects was based on one of the Physware final projects.

This course was the first in a series of workshops designed to enhance the quality of physics education at the tertiary level in the developing world. Its organization was based on a recommendation of the physics education task force of the World Conference on Physics and Sustainable Development (WCPSD). This 2005 Conference was cosponsored by ICTP, the International Union of Pure and Applied Physics (IUPAP), UNESCO and the South African Institute of Physics.

Interest in this first course was so high that the course DirectorsPratibha Jolly (University of Delhi, India), Priscilla Laws (Dickinson College, U.S.), Elena Sassi (University of Naples, “Federico II”, Italy), and Dean Zollman (Kansas State University, U.S.) - were about to choose 32 outstanding participants from 26 countries from a pool of over 200 applicants.

The participants did hands-on work in collaborative groups of two to four. During the first week, the focus was on constructing measuring tools using materials that could be easily found in developing countries. Devices such as pendulum timers constructed using vines, wire, string, fishing line and fibers along with bobs made of nuts, berries, balls, and clay. Length measures were established by using a stick and multiples of it, etc. Coins were used as masses as well as rolling and

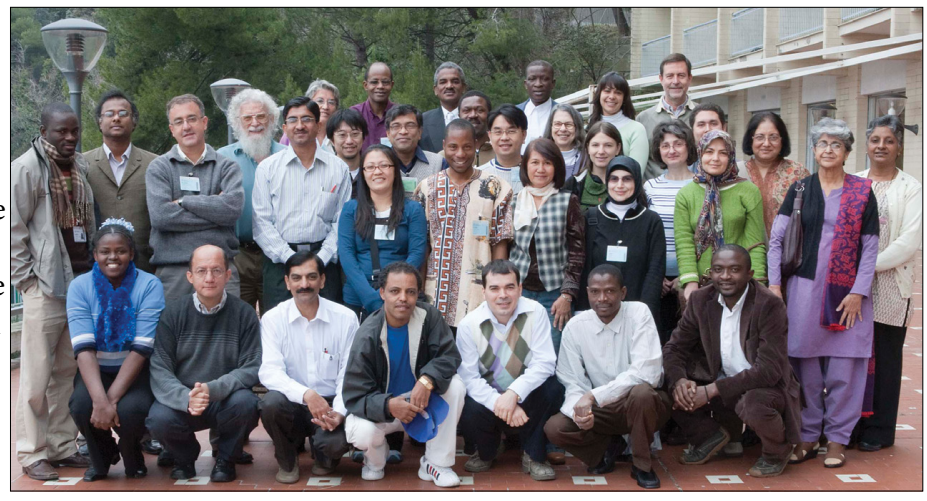

Fig. 1. Physware group photo.

sliding objects. We discovered that "g" is extremely hard to measure directly without electronic technology or the introduction of some of Galileo's famous tricks.

In the second week, we turned our attention to basic mechanics experiments using digital watches and computers interfaced with motion detectors and force probes. Mobile phones equipped with video cameras are ubiquitous in Latin America and are spreading throughout Africa. Several of our week-two projects involved motion capture and analysis using video clips.

One of the most exciting outcomes of the Physware course was that a participant from Latin America set up a blog for the entire group. So we are still communicating, and each year I get many Happy New Year messages from all over the world. The directors are making good progress on getting the support needed to offer regional and international Physware courses on a full range of introductory physics topics. 